\title{
Clinical Utility of Arterial Spin-Labeling as a Confirmatory Test for Suspected Brain Death
}

\author{
K.M. Kang, T.J. Yun, B.-W. Yoon, B.S. Jeon, S.H. Choi, J.-h. Kim, J.E. Kim, C.-H. Sohn, and M.H. Han
}

\begin{abstract}
SUMMARY: Diagnosis of brain death is made on the basis of 3 essential findings: coma, absence of brain stem reflexes, and apnea. Although confirmatory tests are not mandatory in most situations, additional testing may be necessary to declare brain death in patients in whom results of specific components of clinical testing cannot be reliably evaluated. Recently, arterial spin-labeling has been incorporated as part of MR imaging to evaluate cerebral perfusion. Advantages of arterial spin-labeling include being completely noninvasive and providing information about absolute CBF. We retrospectively reviewed arterial spin-labeling findings according to the following modified criteria based on previously established confirmatory tests to determine brain death: 1) extremely decreased perfusion in the whole brain, 2) bright vessel signal intensity around the entry of the carotid artery to the skull, 3) patent external carotid circulation, and 4) "hollow skull sign" in a series of 5 patients. Arterial spin-labeling findings satisfied the criteria for brain death in all patients. Arterial spin-labeling imaging has the potential to be a completely noninvasive confirmatory test to provide additional information to assist in the diagnosis of brain death.
\end{abstract}

ABBREVIATION: $A S L=$ arterial spin-labeling

B rain death is defined as irreversible loss of brain and brain stem function. The diagnosis of brain death is made on the basis of 3 essential findings: coma, absence of brain stem reflexes, and apnea. ${ }^{1}$ Sometimes it is very difficult to make the clinical diagnosis, however, due to confounding factors such as uncertain reliability of neurologic examination or an inability to use the apnea test. ${ }^{2}$ For example, patients with trauma to the eyes or ears, neuromuscular paralysis, or heavy sedation may not be amenable to appropriate neurologic assessment. In addition, patients who are carbon dioxide retainers cannot undergo an apnea test. ${ }^{3}$ In these cases, additional confirmatory tests are helpful to determine the diagnosis. Confirmatory tests for brain death can be divided into those that demonstrate loss of bioelectrical activity and those that show absence of $\mathrm{CBF}$. As a test to demonstrate the loss of

Received August 15, 2014; accepted after revision November 11.

From the Institute of Radiation Medicine (K.M.K., T.J.Y., S.H.C., J.-h.K., C.-H.S., M.H.H.), Seoul National University Medical Research Center, Seoul, Republic of Korea; Departments of Radiology (K.M.K., T.J.Y., S.H.C., J.-h.K., C.-H.S., M.H.H.), Neu rology (B.-W.Y., B.S.J.), and Neurosurgery (J.E.K.), and Clinical Research Center for Stroke (B.-W.Y., B.S.J.), Clinical Research Institute, Seoul National University Hospital, Seoul, Republic of Korea.

Please address correspondence to Tae Jin Yun, MD, Department of Radiology, Seoul National University Hospital, 101, Daehangno, Jongno-gu, Seoul 110-744, Korea; e-mail: radiologyyun@gmail.com

- Indicates open access to non-subscribers at www.ajnr.org

三 Indicates article with supplemental on-line table.

http://dx.doi.org/10.3174/ajnr.A4209 bioelectrical activity, electroencephalography is used in many countries and remains one of the most well-validated confirmatory tests. ${ }^{1}$ Although electroencephalography is sensitive to hypothermia, drugs, or extreme hypotension and confounding artifacts can appear in the environment of the intensive care unit because of the presence of multiple devices, this technique is still used extensively as a confirmatory test to diagnose brain death. ${ }^{1,2}$

Another category of confirmatory test involves demonstrating the absence of CBF. ${ }^{3}$ Bernat $^{4}$ emphasized that the surest way to demonstrate irreversible global loss of clinical brain function is to show complete absence of intracranial blood flow. Cerebral angiography was the first technique used to demonstrate the absence of intracranial circulation distal to the intracranial portions of the internal carotid and vertebral arteries in brain death. ${ }^{5}$ Other techniques used to determine the absence of CBF include cerebral intravenous DSA, intravenous radionuclide angiography, SPECT, echoencephalography, measurement of arm-to-retina circulation time, ophthalmic artery pressure measurement, xenon-enhanced CT, MRA, CTA, CT perfusion, and transcranial Doppler sonography. ${ }^{6}$ The preferred confirmatory test for $\mathrm{CBF}$ is angiography or a radiotracer method. ${ }^{2,3}$ However, cerebral angiography is invasive with the risk of vessel injury. Furthermore, both angiography and radiotracer methods require not only an injection of exogenous material such as contrast media or radioisotopes but also additional exposure to radiation.

Arterial spin-labeling (ASL) is a noninvasive MR imaging 


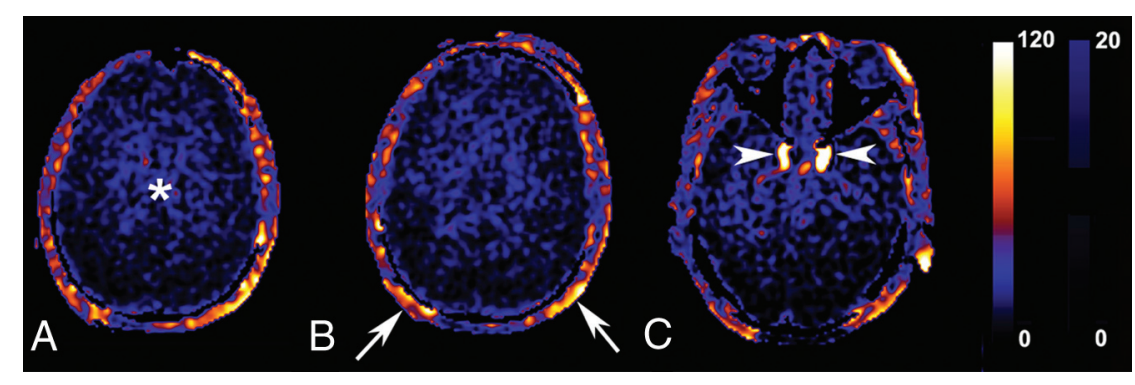

FIG 1. Representative arterial spin-labeling images for each criterion: extremely decreased perfusion in the whole brain (asterisk, A); patent external carotid circulation and the hollow skull sign (arrows, $B$ ), and bright vessel signal intensity around the entry of the carotid artery to the skull (arrows, C). The darker signal intensity in the bilateral parieto-occipital lobes seems to be due to the long transit time exceeding that of the postlabeling decay. Scale units: $\mathrm{mL} \times 100 \mathrm{~g}^{-1} \times$ $\min ^{-1}$.

method to evaluate cerebral perfusion that does not require injection of any exogenous contrast agent or tracers. ${ }^{7}$ Instead, it uses endogenous arterial water labeled by radiofrequency pulses as a freely diffusible tracer. Therefore, there is no radiation exposure with ASL. In addition, ASL is beneficial in terms of resource allocation compared with DSC or radionuclide scans because it has lower cost and is time-efficient. Furthermore, because ASL can provide information regarding CBF values noninvasively, it is quite appropriate for the unique clinical setting of suspected brain death, in which the patient's family frequently refuses any additional invasive examinations. One case has been previously reported on the use of ASL to evaluate brain death. ${ }^{8}$ The purpose of this study was to investigate the potential of ASL as a confirmatory test to determine brain death.

\section{MATERIALS AND METHODS \\ Case Series}

This retrospective study was approved by our institutional review board. Informed consent was waived. After reviewing our radiology data base from June 2010 to April 2014, we identified 5 patients with clinically diagnosed brain death who underwent ASL MR imaging. These 5 patients ( 3 men and 2 women; median age, 60 years; range, $48-75$ years) form the basis of this study, though a case report based on 1 of these 5 subjects was published previously. ${ }^{8}$ The brain deaths occurred following the return of spontaneous circulation after cardiac arrest $(n=2)$, large cerebral infarction $(n=1)$, encephalitis $(n=1)$, and systemic lupus erythematous encephalopathy $(n=1)$.

The diagnosis of brain death was made on the basis of 3 clinical findings: coma (with a known cause), absence of brain stem reflexes, and apnea. ${ }^{2}$ These 3 clinical findings need to be present to make the definitive diagnosis. The brain death diagnosis was made by at least 2 neurologists according to criteria established by local law. The prerequisites to determine brain death were an irreversible and proximate cause of coma; deep coma without spontaneous respiration; and no evidence of a neurologic state that could mimic brain death, including drug intoxication or poisoning, use of sedatives, metabolic or endocrine abnormalities, hypothermia, and shock. Furthermore, there was no return of the above neurologic functions after 6 hours of observation. In addition, all patients showed generalized continuous low-voltage background activity on electroencephalography.
All patients were clinically brain dead before acquisition of MR imaging. Even though the patients were already diagnosed as being brain dead by clinical criteria, the physicians requested MR imaging to determine the irreversible cause of coma and to evaluate the features of brain parenchyma, intracranial arteries, and perfusion. The mean interval between ASL imaging and brain death in the 5 patients was 3 days (range, 1-7 days). All patients underwent MR imaging on a $1.5 \mathrm{~T}$ unit (Signa HDTx; GE Healthcare, Milwaukee, Wisconsin) by using an 8-channel head coil to acquire ASL perfusion MR imaging. MR imaging sequences including T1WI, T2WI, FLAIR, DWI (b factors, 0 and $1000 \mathrm{~mm} / \mathrm{s}^{2}$ ), and ASL were acquired. The ASL protocol applied was as follows: ASL sequence parameters: TR, $4225 \mathrm{~ms}$; TE, $9.9 \mathrm{~ms}$; FOV , $24 \times 24 \mathrm{~cm}^{2}$; section thickness, $5 \mathrm{~mm}$; NEX, 3 ; and number of interleaved sections, 32 . Pseudocontinuous spin-labeling for 1.5 seconds before a post-spin-labeling delay of 1.5 seconds was applied. Three of the 5 patients underwent 3D TOF MRA, whereas 2 patients underwent contrast-enhanced MRA with subsequent maximum-intensity-projection reconstruction during the MR imaging examination. Specific parameters for MR imaging sequences are provided in the On-line Table.

Two neuroradiologists (K.M.K. and T.J.Y., with 6 and 12 years of experience in neuroradiology, respectively) reviewed the ASL images with consensus. The ASL findings for diagnosing the absence of CBF were made on the basis of the findings from previous studies by using reference standard tests, radionuclide scans, and cerebral angiography. ${ }^{3,9-16}$ The ASL findings used to assess the absence of intracranial blood flow were the following: 1) extremely decreased perfusion in the whole brain, 2) bright vessel signal intensity around the entry of the carotid artery to the skull suggesting flow stagnation, and 3) patent external carotid circulation corresponding to the findings of brain death on conventional angiography. ${ }^{3}$ In addition, we reviewed the "hollow skull sign," which was defined as a finding of extremely impaired cerebral perfusion with preserved perfusion in the distribution of the external carotid artery on ASL images. The finding was modified from the hollow skull sign described in scintigraphy for the diagnosis of brain death. ${ }^{10,12,16,17}$

Figure 1 shows representative images of each criterion.

All images were spatially normalized to the ethnicity-specific T1 template before determining CBF by using SPM5 software (http://www.fil.ion.ucl.ac.uk/spm/software/spm5) and custom scripts in Matlab 7.6.0, R2008 (MathWorks, Natick, Massachusetts). CBF values were measured in ethnicity-specific volume of interest for whole-brain parenchyma by a neuroradiologist (T.J.Y., with 12 years of experience in neuroradiology). ${ }^{18-20}$

Extremely impaired perfusion was detected in the whole brain in all 5 cases. In addition, bright signal intensity was observed at the cavernous or petrous ICA levels in all cases. The bright signal intensity occurred at the unilateral petrous ICA in 2 patients and at the bilateral cavernous or petrous ICAs in 3 patients. In contrast, perfusion supplied by the external carotid artery branches 


\begin{tabular}{|c|c|c|c|c|c|c|c|c|c|}
\hline \multirow[b]{2}{*}{ Case } & \multirow[b]{2}{*}{$\begin{array}{l}\text { Age } \\
\text { (yr) }\end{array}$} & \multirow[b]{2}{*}{ Sex } & \multirow[b]{2}{*}{ Causes } & \multicolumn{4}{|c|}{ ASL Findings } & \multirow[b]{2}{*}{$\begin{array}{c}\text { CBF } \\
(\text { Mean } \pm \text { SD, } \\
\left.\mathrm{mL} \times 100 \mathrm{~g}^{-1} \times \mathrm{min}^{-1}\right)\end{array}$} & \multirow[b]{2}{*}{ MRA } \\
\hline & & & & $\begin{array}{l}\text { Extremely Decreased } \\
\text { Perfusion in the } \\
\text { Whole Brain }\end{array}$ & $\begin{array}{l}\text { Bright Vessel Signal Intensity } \\
\text { around the Entry of Carotid } \\
\text { Artery to the Skull } \\
\text { ( } \pm \text { Level) }\end{array}$ & $\begin{array}{l}\text { External } \\
\text { Carotid } \\
\text { Circulation }\end{array}$ & $\begin{array}{l}\text { Hollow } \\
\text { Skull } \\
\text { Sign }\end{array}$ & & \\
\hline 1 & 71 & M & Cardiac arrest & + & + , Rt petrous ICA & + & + & $10.0 \pm 2.3$ & NV \\
\hline 2 & 60 & M & Cardiac arrest & + & + , Bilateral cavernous ICAs & + & + & $8.5 \pm 3.2$ & NA \\
\hline 3 & 75 & $\mathrm{~F}$ & Cerebral infarction & + &,+ Lt petrous ICA & + & + & $7.0 \pm 4.5$ & NV \\
\hline 4 & 50 & M & Encephalitis & + & + , Rt cavernous ICA and Lt petrous ICA & + & + & $7.5 \pm 6.4$ & NV \\
\hline 5 & 48 & $\mathrm{~F}$ & SLE encephalopathy & + & + , Rt petrous ICA and Lt cavernous ICA & + & + & $6.9 \pm 2.8$ & NA \\
\hline
\end{tabular}

Note:-Lt indicates left; NA, not available; NV, no visualization of intracranial arterial flow; Rt, right; SLE, systemic lupus erythematous; +, present.

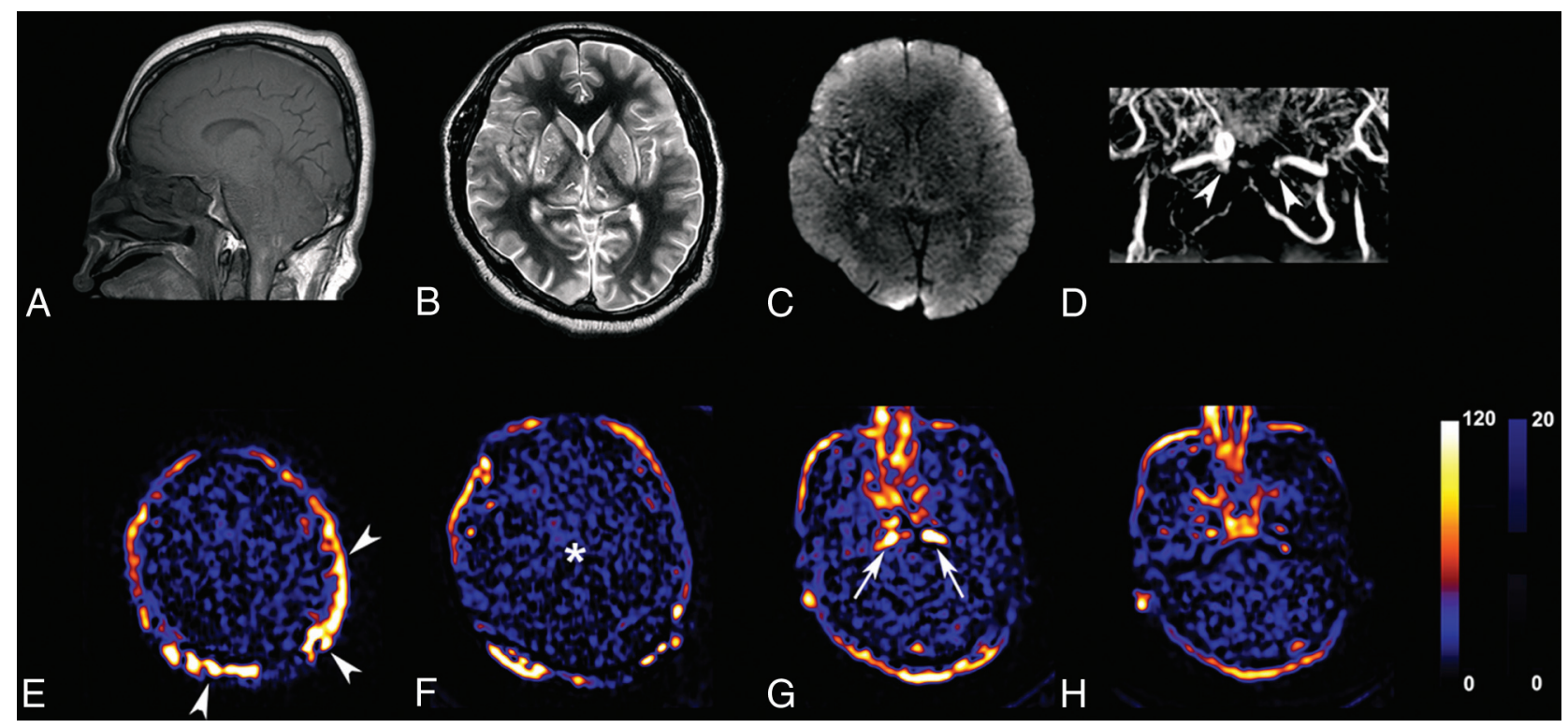

FIG 2. Representative MR imaging and arterial spin-labeling images in a 50-year-old man with brain death. Sagittal TTWI (A) shows tonsillar impaction in the foramen magnum. Axial T2WI (B) reveals gyral swelling with decreased ventricle size, and DWI (C) shows diffuse hyperintensity involving the whole brain. Contrast-enhanced MRA $(D)$ shows an abrupt cutoff in the bilateral ICAs around the entries of carotid arteries to the skull (arrowheads, $D$ ) and the absence of intracranial arterial flow. The ASL images $(E-H)$ satisfy all ASL criteria supporting brain death: extremely decreased perfusion in the whole brain (asterisk, F), bright signal intensity in the ICA around the entry of the carotid artery into the skull suggesting flow stagnation (arrows, $G$ ), and patent external carotid circulation and the hollow skull sign (arrowheads, E). Scale units: $\mathrm{mL} \times 100 \mathrm{~g}^{-1} \times \mathrm{min}^{-1}$.

was normal in all cases. In addition, the hollow skull sign was seen in all patients on ASL. No intracranial arterial flow was detected on MRA in 3 patients (Table). The CBF values for the whole-brain parenchyma ranged from 6.9 to $10.0 \mathrm{~mL} \times 100 \mathrm{~g}^{-1} \times \mathrm{min}^{-1}$, with a mean value of $8.0 \pm 1.3 \mathrm{~mL} \times 100 \mathrm{~g}^{-1} \times \mathrm{min}^{-1}$, and all CBF values were $<15 \mathrm{~mL} \times 100 \mathrm{~g}^{-1} \times \mathrm{min}^{-1}$ (Table). Representative MR images, including ASL in patients who were brain dead, are shown in Figs 2 and 3. ASL and MRA images of a healthy subject and a patient who was brain dead or with cardiac arrest are shown in Fig 4.

\section{DISCUSSION}

Brain death is a clinical diagnosis based on neurologic assessment and confirmatory tests conducted according to the situation. In our study, all brain death cases showed severe compromise in the intracranial circulation and patent external carotid artery perfusion. Very bright vessel signal intensity in the ICA around the entry of carotid artery to the skull without signal intensity in the intracerebral circulation was also observed. This "bright vessel signal" represents labeled blood with a notable transit delay, presumably secondary to elevated intracranial pressure, especially when relatively normal external carotid artery blood flow is seen. This finding corresponds to visualizing the extracranial ICA with no visualization of the anterior circulation beyond the level of the supraclinoid ICAs on conventional angiography. ${ }^{3}$ In cases of patients showing unilateral bright vessel signals, there had been chronic occlusion in the contralateral ICA. Therefore, we assume that the bright vessel signal around the entry of the carotid artery to the skull might not be seen in cases of chronic stenoses in the bilateral ICAs.

According to the criteria we used, ASL revealed the absence of $\mathrm{CBF}$ in brain death by both visual and quantitative analysis. Because the absence of CBF is generally accepted as a definite sign of brain death, ${ }^{3,5,11,12,16}$ ASL has the potential to be a noninvasive and reliable confirmatory test for diagnosing brain death. However, the assessment of criteria should be performed carefully and strictly. If either the absence of intracranial flow or the preservation of extracranial flow is not observed, then the case should not be diagnosed as brain death. We had a 20 -year-old female patient who experienced cardiac arrest during an MR imaging examination. Because the patient had a myocardial infarction after acqui- 


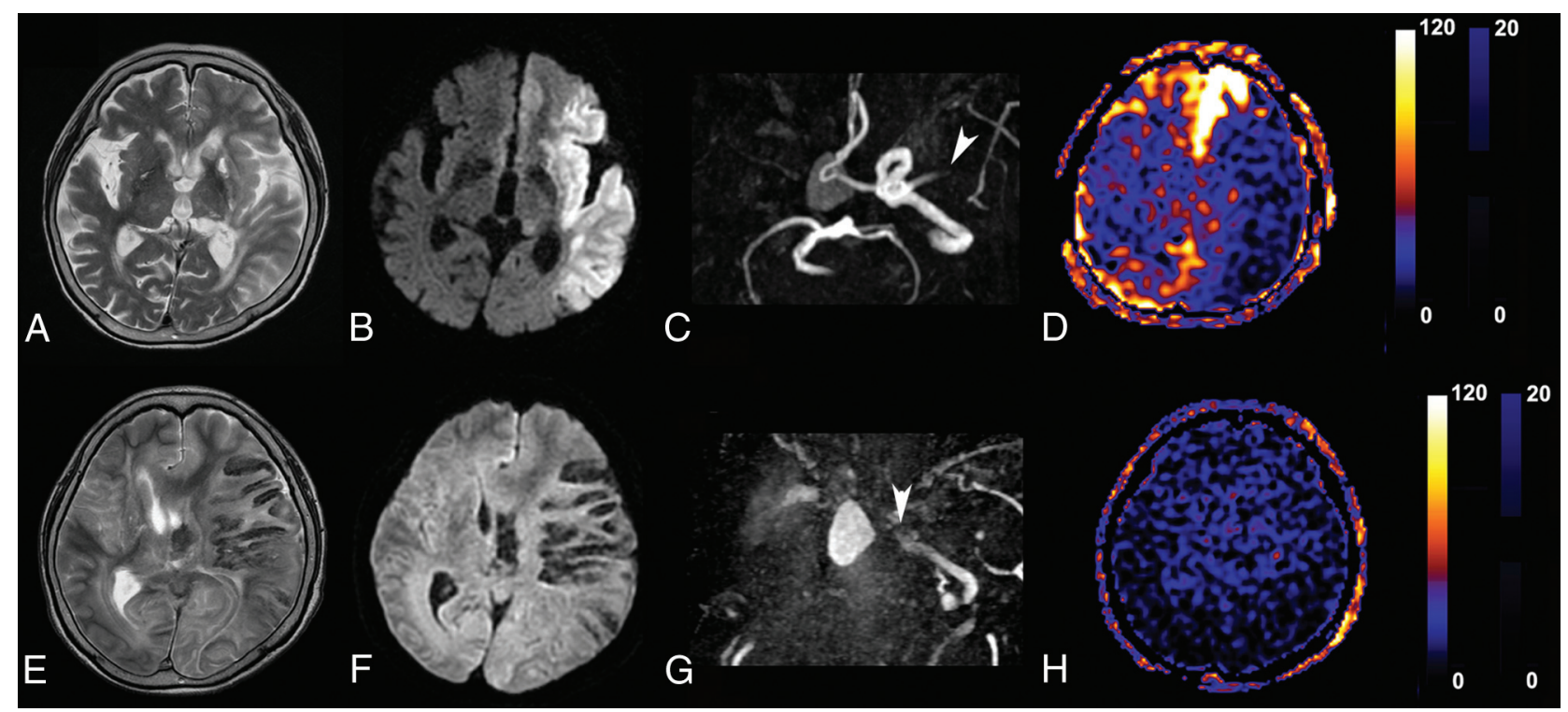

FIG 3. Representative arterial spin-labeling images in a 75-year-old woman with brain death following an infarct. The images in the upper row were acquired when the patient was admitted with an infarct. The images in the lower row were acquired when the patient was declared brain dead. T2WI (A) shows hyperintense parenchymal swelling in the left MCA territory, and DWI $(B)$ demonstrates increased signal intensity in the corresponding area. TOF MRA (C) reveals total occlusion in the left proximal MCA (arrowhead). The patient also had chronic stenosis in the right proximal ICA. Although cerebral perfusion is severely impaired in the left MCA territory on the ASL image (D), the CBF values in other areas of the brain are preserved. T2WI $(E)$ and DWI $(F)$ performed shortly after the diagnosis of brain death depict diffuse gyral swelling and diffusion hyperintensity, respectively, in the whole brain. TOF MRA $(G)$ shows an abrupt cutoff of the left distal ICA (arrowhead) and absence of intracranial vessels. ASL image $(H)$ demonstrates markedly decreased CBF in the whole brain. Scale units: $\mathrm{mL} \times 100 \mathrm{~g}^{-1} \times \mathrm{min}^{-1}$.

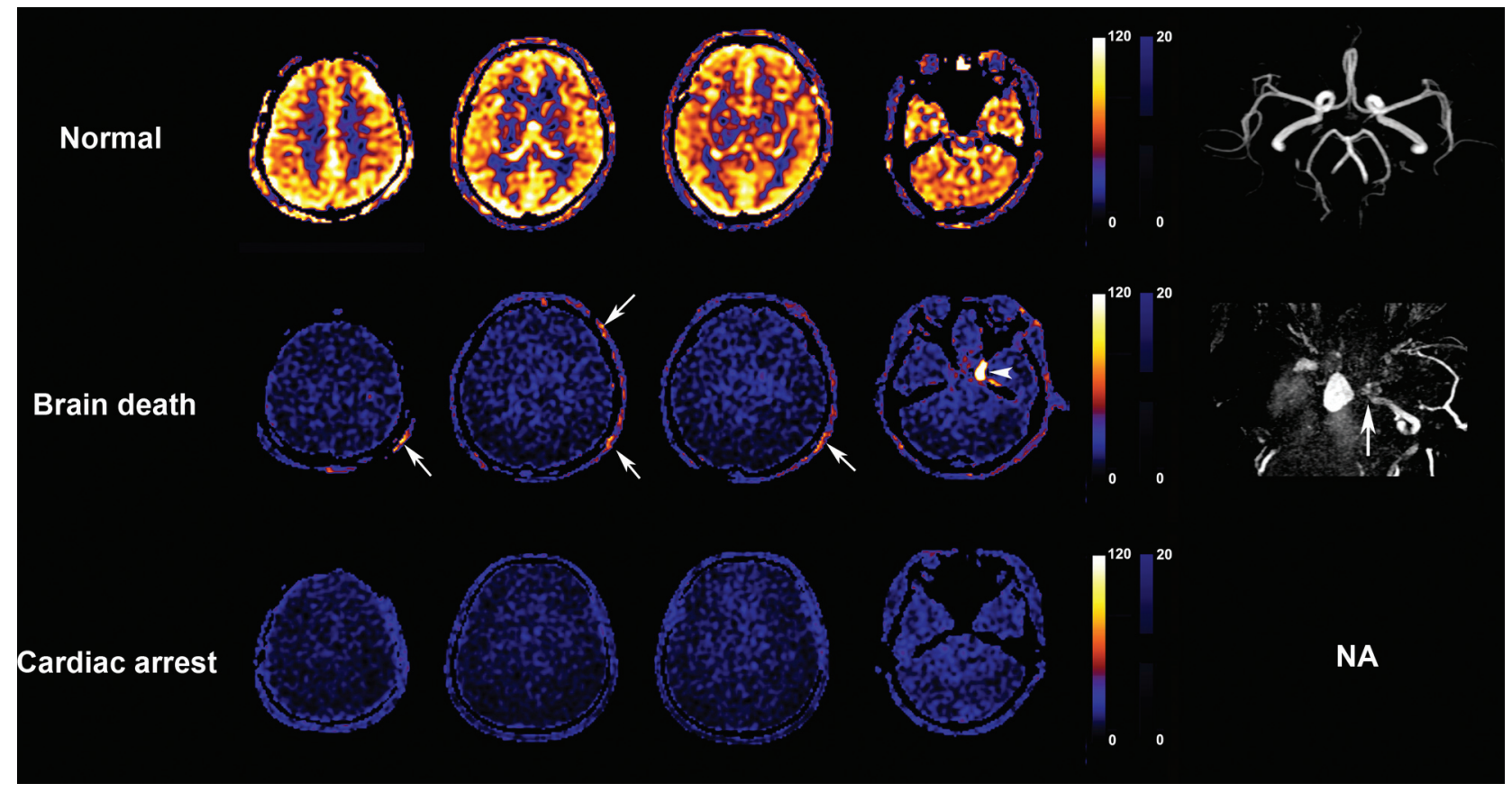

FIG 4. Representative arterial spin-labeling images in a healthy subject, a patient with brain death, and a patient who had cardiac arrest during an MR imaging examination. These figures show representative ASL and MRA images in a 46-year-old woman as a healthy subject (upper row), a 75-year-old woman with brain death (middle row), and a 20-year-old woman who had sudden cardiac arrest just before the acquisition of ASL images during an MR imaging examination (bottom row). ASL images from the patient with brain death show extremely impaired brain perfusion in the whole brain, bright vessel signal intensity around the level of entry of the carotid artery to the skull (arrowhead), and patent external carotid circulation (arrows) compared with the ASL images from the healthy subject. An MRA image in the patient with brain death (right column of middle row) shows an abrupt cutoff in the left distal ICA (arrow). Although the ASL images from the patient with cardiac arrest also show extremely impaired brain perfusion in the whole brain, there is neither bright vessel signal intensity around the entry of carotid artery to the skull nor patent external carotid circulation. Even though TOF MRA acquired just before the cardiac arrest had normal findings, acquisition of contrast-enhanced MRA after cardiac arrest failed. Scale units: $\mathrm{mL} \times 100 \mathrm{~g}^{-1} \times \mathrm{min}^{-1}$. NA indicates not available. 
sition of TOF intracranial MRA, the intracranial MRA findings were normal. However, ASL images acquired immediately after the myocardial infarction showed extremely impaired extracranial flow and extremely decreased CBF calculated at $8.62 \mathrm{~mL} \times$ $100 \mathrm{~g}^{-1} \times \mathrm{min}^{-1}$, resulting from severely impaired cardiac output (Fig 4). The ASL findings of this patient and patients with brain death were different in that external carotid artery perfusion was always preserved in brain death. In addition, insufficient labeling of arterial flow caused by atherosclerosis or stent placement at the level of labeling might prevent demonstrating signals for both intracranial and extracranial flow on ASL. Therefore, both intracranial circulatory cessation and preserved external carotid artery perfusion are necessary to determine the cessation of intracranial flow in cases of brain death.

In contrast to other perfusion techniques, including dynamic susceptibility contrast MR perfusion and SPECT, ASL provides an absolute CBF value. In the present study, the CBF values in the whole brain parenchyma ranged from 6.9 to $10.0 \mathrm{~mL} \times 100 \mathrm{~g}^{-1} \times$ $\min ^{-1}$. Even though there is no established threshold or CBF cutoff that defines brain death, according to several reports, minimal CBF in viable brain is $15 \mathrm{~mL} \times 100 \mathrm{~g}^{-1} \times \mathrm{min}^{-1} \cdot{ }^{21-25} \mathrm{Al}-$ though quantifying CBF with ASL could help reveal the lack of $\mathrm{CBF}$ in cases of brain death, a postlabel delay of 1.5 seconds used in our study might be relatively short in the setting of markedly prolonged transit. $^{26,27}$ In such cases, one should use caution in applying absolute quantitative $\mathrm{CBF}$ values across practice settings. Using a multidelay longer postlabel decay time may be helpful in overcoming this limitation. ${ }^{26,27}$ Furthermore, there is no welldefined threshold or CBF cutoff that defines irreversible and/or definite brain death. Future work may further explore such a possible threshold.

In our study, we observed the hollow skull sign with ASL in all patients with brain death. The hollow skull sign was originally a scintigraphic finding to confirm brain death. ${ }^{12}$ Now, it serves as a secondary sign of brain death. Among scintigraphic examinations for brain death, SPECT imaging provides information additional to that of planar imaging. ${ }^{18}$ SPECT imaging has some benefit for differentiating scalp, parotid, or neck muscle uptake from brain uptake. In addition, evaluation of the posterior fossa is better with SPECT imaging than with planar imaging because of its tomographic nature. Furthermore, ASL provides not only the same advantages as SPECT without injecting exogenous material, it also has other potential advantages such as resource allocation, lower cost, and time-efficiency compared with DSC and radionuclide scans. Therefore, ASL seems to be a promising method to replace scintigraphic methods.

The concept of brain death is often difficult for families to accept when dealing with a tragic loss. Grieving family members are understandably occasionally against any invasive test to confirm brain death. Hence, easily applied clinical tools that help a patient's kin understand brain death are needed. In our study, we introduced 4 ASL findings to determine brain death. These findings are fairly straightforward features to interpret. We think that applying ASL in cases of suspected brain death can help family members accept the grave condition of the patient so that they might be approached about possible organ donation before organ viability becomes a concern.
Our study had several limitations. First, there is selection bias because this is a clinical report for a case series of patients with known brain death. Future studies need to be performed prospectively to determine diagnostic accuracy and clinical effectiveness. Second, only a limited number of patients with brain death were evaluated. Thus, we could not perform a statistical analysis to investigate the diagnostic accuracy of ASL to determine brain death. Perfect diagnostic accuracy is an essential prerequisite as a brain death diagnostic tool because the presence of a false-positive case would be unacceptable. Further studies with a larger sample size and a prospective design are required to validate whether ASL imaging could be used to determine brain death. Third, ASL requires transfer of a critically ill patient to the MR imaging scanner. Its lack of portability is a major limitation of ASL as a confirmatory test for brain death. Finally, a postlabel delay of 1.5 seconds used in our study might be relatively short in the setting of severely impaired cerebral perfusion.

\section{CONCLUSIONS}

ASL imaging has the potential to serve as a noninvasive confirmatory test to provide additional information to assist in the diagnosis of brain death.

\section{ACKNOWLEDGMENTS}

We would like to thank So Young Yun (Seoul National University Hospital, Seoul, South Korea), who assisted with recruitment of the subjects.

Disclosures: Tae Jin Yun-RELATED: Grant: National Research Foundation of Korea (NRF-2013R1A1A2008332).* *Money paid to the institution.

\section{REFERENCES}

1. Wijdicks EF. The diagnosis of brain death. N Engl J Med 2001; 344:1215-21

2. Wijdicks EF, Varelas PN, Gronseth GS, et al. Evidence-based guideline update: determining brain death in adults—report of the Quality Standards Subcommittee of the American Academy of Neurology. Neurology 2010;74:1911-18

3. Heran MK, Heran NS, Shemie SD. A review of confirmatory tests in evaluating brain death. Can J Neurol Sci 2008;35:409

4. Bernat JL. On irreversibility as a prerequisite for brain death determination. Adv Exp Med Biol 2004;550:161-67

5. Bücheler E, Käufer C, Düx A. Cerebral angiography to determine brain death [in German]. Fortschr Geb Rontgenstr Nuklearmed 1970; 113:278-96

6. Machado C. Diagnosis of brain death. Neurol Int 2010;2:e2

7. Wolf RL, Detre JA. Clinical neuroimaging using arterial spin-labeled perfusion magnetic resonance imaging. Neurotherapeutics 2007;4:346-59

8. Yun TJ, Sohn CH, Yoon BW, et al. Brain death evaluation of cerebral blood flow by use of arterial spin labeling. Circulation 2011;124: 2572-73

9. Bradac G, Simon R. Angiography in brain death. Neuroradiology 1974;7:25-28

10. Abdel-Dayem HM, Bahar RH, Sigurdsson GH, et al. The hollow skull: a sign of brain death in Tc-99m HM-PAO brain scintigraphy. Clin Nucl Med 1989;14:912-16

11. Wieler H, Marohl K, Kaiser K, et al. Tc-99m HMPAO cerebral scintigraphy: a reliable, noninvaslve method for determination of brain death. Clin Nucl Med 1993;18:104-09

12. Orrison WW Jr, Champlin AM, Kesterson OL, et al. MR 'hot nose sign' and 'intravascular enhancement sign' in brain death. AJNR Am J Neuroradiol 1994;15:913-16

AJNR Am J Neuroradiol 36:909-14 May 2015 www.ajnr.org 913 
13. Bonetti M, Ciritella P, Valle G, et al. 99mTc HM-PAO brain perfusion SPECT in brain death. Neuroradiology 1995;37:365-69

14. Ishii $\mathrm{K}$, Onuma T, Kinoshita T, et al. Brain death: MR and MR angiography. AJNR Am J Neuroradiol 1996;17:731-35

15. Qureshi AI, Kirmani JF, Xavier AR, et al. Computed tomographic angiography for diagnosis of brain death. Neurology 2004;62:652-53

16. Sinha P, Conrad GR. Scintigraphic confirmation of brain death. Semin Nucl Med 2012;42:27-32

17. Al-Shammri S, Al-Feeli M. Confirmation of brain death using brain radionuclide perfusion imaging technique. Med Princ Pract 2004; 13:267-72

18. Lee JS, Lee DS, Kim J, et al. Development of Korean standard brain templates. J Korean Med Sci 2005;20:483-88

19. Collins DL, Neelin P, Peters TM, et al. Automatic 3D intersubject registration of MR volumetric data in standardized Talairach space. J Comput Assist Tomogr 1994;18:192-205

20. Yun TJ, Sohn $\mathrm{CH}$, Han MH, et al. Effect of carotid artery stenting on cerebral blood flow: evaluation of hemodynamic changes using arterial spin labeling. Neuroradiology 2013;55:271-81

21. Powers WJ, Grubb RL, Darriet D, et al. Cerebral blood flow and cerebral metabolic rate of oxygen requirements for cerebral function and viability in humans. J Cereb Blood Flow Metab 1985;5:600-08
22. Latchaw RE, Yonas H, Hunter GJ, et al. Guidelines and recommendations for perfusion imaging in cerebral ischemia: a scientific statement for healthcare professionals by the Writing Group on Perfusion Imaging, from the Council on Cardiovascular Radiology of the American Heart Association. Stroke 2003;34:1084-104

23. Astrup J. Energy-requiring cell functions in the ischemic brain: their critical supply and possible inhibition in protective therapy. J Neurosurg 1982;56:482-97

24. Schuier F, Hossmann K. Experimental brain infarcts in cats. II. Ischemic brain edema. Stroke 1980;11:593-601

25. Jones TH, Morawetz RB, Crowell RM, et al. Thresholds of focal cerebral ischemia in awake monkeys. J Neurosurg 1981;54:773-82

26. Qiu D, Straka M, Zun Z, et al. CBF measurements using multidelay pseudocontinuous and velocity-selective arterial spin labeling in patients with long arterial transit delays: comparison with xenon CT CBF. J Magn Reson Imaging 2012;36:110-19

27. Alsop DC, Detre JA, Golay X, et al. Recommended implementation of arterial spin-labeled perfusion MRI for clinical applications: a consensus of the ISMRM perfusion study group and the European consortium for ASL in dementia. Magn Reson Med 2014 Apr 8. [Epub ahead of print] 